\title{
p53 protein expression in patients with myelodysplasia treated with allogeneic bone marrow transplantation
}

\author{
ACHILLE PICH $^{1}$, LAURA GODIO ${ }^{2}$ and LAURA DAVICO BONINO ${ }^{2}$ \\ ${ }^{1}$ Department of Molecular Biotechnology and Health Sciences, Section of Pathology, University of Turin; \\ ${ }^{2}$ Azienda Ospedaliera Città della Salute e della Scienza, Anatomia e Istologia Patologica 1U, I-10126 Turin, Italy
}

Received November 17, 2016; Accepted February 14, 2017

DOI: $10.3892 / \mathrm{mco} .2017 .1225$

\begin{abstract}
Tumor protein 53 mutations adversely affect the prognosis of myelodysplastic syndromes (MDS); however, few studies have reported on the prognostic significance of the expression of $\mathrm{p} 53$ protein in MDS. The current study investigated p53 immunoreactivity (p53-IR) in bone marrow biopsies (BMBs) obtained at diagnosis from 18 patients ( 6 females and 12 males; mean age, 50.5 years) with MDS that underwent bone marrow transplantation (BMT) to determine the associations between clinical and histopathological data and outcome. There were 5 refractory cytopenia with multilineage dysplasia (RCMD) and 13 refractory anemia with excess blasts, type 2 (RAEB-2) cases. p53-IR was assessed as the percentage of hematopoietic cells exhibiting intense nuclear staining. The cut off for positivity was $5 \%$ of stained cells. A positive p53-IR was detected in 7 patients $(38.9 \%)$ and was associated with age $(\mathrm{P}=0.005)$ and pattern of $\mathrm{BM}$ fibrosis $(\mathrm{P}=0.03)$. A positive p53-IR was more frequent in females, in highly cellular BMBs and in RAEB-2 cases. Overall survival (OS) was associated with patients' age $(\mathrm{P}=0.01)$, hemoglobin level $(\mathrm{P}=0.04)$, type of $\operatorname{MDS}(\mathrm{P}=0.05)$, degree of $\mathrm{BM}$ fibrosis $(\mathrm{P}=0.006)$ and number of $\mathrm{BM}$ blasts $(\mathrm{P}=0.05)$. The $\mathrm{OS}$ of patients with negative p53-IR tended to be longer compared with that of patients with positive p53-IR, although this difference was not statistically significant $(\mathrm{P}=0.1)$. Despite the limitation of the low number of cases, the present results indicate that a positive p53-IR at diagnosis is associated with clinically more aggressive MDS subtypes and adverse histological prognostic factors, such as BM fibrosis. Therefore, the evaluation of p53 expression of BMBs of patients with MDS may be introduced in the histopathological work-up of the disease.
\end{abstract}

Correspondence to: Professor Achille Pich, Department of Molecular Biotechnology and Health Sciences, Section of Pathology, University of Turin, Via Santena 7, I-10126 Turin, Italy

E-mail: achille.pich@unito.it

Key words: myelodysplastic syndromes, TP53 mutations, immunohistochemistry, prognosis

\section{Introduction}

Myelodysplastic syndromes (MDS) are a group of clonal hematological disorders characterized by ineffective hematopoiesis, peripheral cytopenias and an increased risk of development of acute myeloid leukemia (1). A number of chromosomal abnormalities have been detected in $~ 50-60 \%$ of patients with de novo MDS and in $\leq 80 \%$ of patients with therapy-related MDS (2). Among the classical oncogenic abnormalities, those involving the tumor protein 53 (TP53) gene have been extensively investigated in MDS. TP53 mutations were detected primarily in high-risk and therapy-related MDS, frequently in association with complex chromosomal abnormalities $(3,4)$. In such diseases, TP53 mutations were revealed to have a negative prognostic impact $(3,5)$.

The wild-type p53 protein is normally undetectable by immunohistochemistry (IHC), due to its short half-life. By contrast, the majority of mutated proteins have a prolonged half-life, accumulate within the nucleus and can be easily detected in formalin-fixed, paraffin-embedded tissues (6). Thus, the immunohistochemical detection of p53 protein suggests an underlying mutation in the gene (7). It has been previously demonstrated that aberrant nuclear expression of p53 protein is associated with hemizygous p53 deletion in multiple myeloma $(8,9)$ and chronic lymphocytic leukemia $(10)$. In MDS, immunohistochemical staining for TP53 protein in bone marrow trephine biopsies has revealed a strong correlation with TP53 mutation status (11-13).

A large number of previous studies have reported on the strong prognostic significance of the immunohistochemical detection of p53 protein in tumor pathology; however, few studies have evaluated its prognostic value in MDS (14-16), and certain studies primarily focused on low-risk MDS with $\operatorname{del}(5 q)(11,17)$.

In the present study, p53 immunoreactivity (p53-IR) was investigated in bone marrow biopsies (BMBs) of patients with MDS that underwent bone marrow transplantation (BMT), with the aim of determining its association with clinical and histopathological data.

\section{Patients and methods}

A total of 18 patients that were admitted to the Division of Haematology (Città della Salute e della Scienza and University 

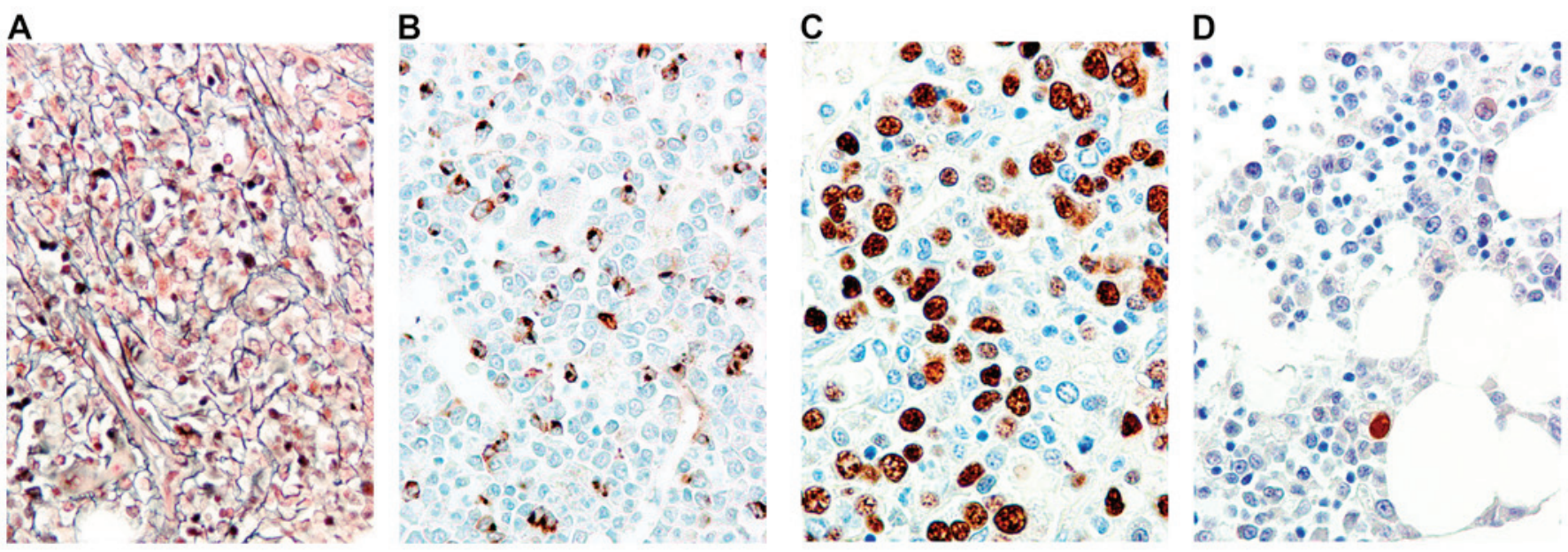

Figure 1. (A) RAEB-2 exhibiting moderate and diffuse reticulin fibrosis (MF-2) (bone marrow biopsy; Gomori stain; magnification, $\mathrm{x} 400$ ). (B) RAEB-2 showing numerous CD34 immunopositive blasts arranged in clusters (bone marrow biopsy; CD34 immunoperoxidase staining; magnification, $\mathrm{x} 400$ ). (C) RAEB-2 in which $>50 \%$ of cells have intensely stained nuclei for p53 (p53-IR positive case; bone marrow biopsy; p53 immunoperoxidase staining; magnification, $x 400$ ). (D) Refractory cytopenia with multilineage dysplasia. Only one myeloid cell shows an intensely p53 stained nucleus (p53-IR negative case) (bone marrow biopsy; p53 immunoperoxidase staining; magnification, x400). RAEB-2, refractory anemia with excess blasts, type 2; p53-IR, p53 immunoreactivity.

of Turin, Italy) from December 1994 to June 2005, with a diagnosis of MDS were retrospectively examined. There were 6 females and 12 males; the mean age was 50.5 years (range, 32-64). Diagnosis of MDS was performed according to the World Health Organization (WHO) criteria (1). In total, 5 patients had refractory cytopenia with multilineage dysplasia (RCMD) and 13 patients had refractory anemia with excess blasts, type 2 (RAEB-2). Hemoglobin level, white blood cell (WBC) and platelet counts were assessed from peripheral blood at diagnosis. All patients underwent allogeneic bone marrow transplantation. General informed consent was obtained according to the guidelines of the Ethics Committee, Città della Salute e della Scienza and University of Turin. Samples were numerically identified, maintaining patients' anonymity.

BM morphology and immunohistochemistry. Serial sections (3- $\mu \mathrm{m}$ thick) from Bouin's solution-fixed, paraffin-embedded BMBs were stained with hematoxylin-eosin, Dominici, Perls and reticulin (Fig. 1A) and immunostained with an automated stainer device (Leica BOND III, Leica Biosystems, Melbourne Pty Ltd, Mount Waverley VIC 3149, Australia) using a polyclonal antibody against myeloperoxidase (cat. no. A0398; Dako; Agilent Technologies, Inc., Santa Clara, CA, USA; dilution, 1:1,000), and monoclonal antibodies against glycophorin A (clone JC159, \#M0819; Dako; Agilent Technologies, Inc., Santa Clara, CA, USA; dilution, 1:50), CD61 (clone 2f2; cat. no. 760-4249; Ventana Medical Systems, Tucson, AZ, USA; undiluted), CD34 (Fig. 1B) (clone QBEnd/10; cat. no. NCL-L-END; Novocastra; Leica Microsystems, Milton Keynes, UK; dilution, 1;50) and p53 (clone DO7, \#NCL-L-p53-DO7; Novocastra; Leica Microsystems, Milton Keynes, UK; dilution, 1:100) at room temperature for $15 \mathrm{~min}$.

Paraffin sections (3- $\mu \mathrm{m}$ thick) on SuperFrost microscope slides were processed with an automated stainer device (Leica BOND III, Leica Microsystems) and pretreated for $30 \mathrm{~min}$ in citrate buffer (pH 6.0; Bond Epitope Retrieval Solution 1, Leica Microsystems). The DO-7 antibody was applied at a 1:100 dilution for $30 \mathrm{~min}$ at room temperature and detected using a Bond Polymer Refine Detection kit (cat. no. DS9800; Leica Microsystems) according to the manufacturer's protocol. The percentage of cells with intense (p53++) nuclear staining was calculated examining $\geq 1,000$ hematopoietic cells at a high magnification ( $\mathrm{x} 40)$ using a standard light microscope. The cut off for positivity (13) was $5 \%$ of stained cells (Fig. 1C and D).

Statistical analysis. The association between p53 immunoreactivity (p53-IR) and clinical or hematological parameters was assessed using one-way analysis of variance and the Fisher's exact test. Univariate survival analyses were based on Kaplan-Meier product-limit estimates of survival distribution, and differences between survival curves were tested using the log-rank test. Overall survival (OS) was calculated from the date of diagnosis to the date of the last observation or death. All analyses were performed using SPSS version 17 (SPSS Inc., Chicago, IL, USA). $\mathrm{P}<0.05$ was considered to indicate a statistically significant difference.

\section{Results}

Association between p53-IR, clinical and hematological features and bone marrow histology. Positive p53-IR was detected in 7/18 (38.9\%) patients. Significant associations were detected between p53-IR and patient age (mean age, 57.3 years for positive p53-IR patients vs. 46.4 years for negative p53-IR patients; $\mathrm{P}=0.005$ ) and the pattern of $\mathrm{BM}$ fibrosis, in which, of the 8 patients with diffuse fibrosis, 6 (75\%) were p53-IR-positive compared with $1(16.7 \%)$ of the 6 patients with focal fibrosis $(\mathrm{P}=0.03)$. A trend toward significance was found for sex, (positive p53-IR in $67 \%$ of females vs. $25 \%$ of males; $\mathrm{P}=0.08$ ), $\mathrm{BM}$ cellularity (mean $\mathrm{BM}$ cellularity, $81.4 \%$ for p53-IR-positive patients vs. $64.5 \%$ for p53-IR-negative patients; $\mathrm{P}=0.1)$ and the degree of $\mathrm{BM}$ fibrosis $(44.4 \%$ of WHO-MF1 and $60 \%$ of WHO-MF2 cases were p53-IR-positive, whereas no WHO-MF0 cases were positive; $\mathrm{P}=0.1)$. No association was found for hemoglobin level, white blood cell 
Table I. Association between p53-IR and clinical and histopathological bone marrow features.

\begin{tabular}{|c|c|c|c|c|}
\hline & & P53-IR positive $(\mathrm{N}=7)$ & P53-IR negative $(\mathrm{N}=11)$ & \\
\hline Variable & $\mathrm{N}$ & Mean \pm SD & Mean \pm SD & P-value ${ }^{a}$ \\
\hline Age, years & 18 & $57.3 \pm 4.6$ & $46.4 \pm 8$ & $<0.01$ \\
\hline $\mathrm{Hb}$ level, g/dl & 18 & $8.38 \pm 1.8$ & $9.06 \pm 1.70$ & 0.40 \\
\hline WBC count, $\times 10^{9} / 1$ & 18 & $3.421 \pm 2.989$ & $4.380 \pm 3.130$ & 0.60 \\
\hline Plt count, $\mathrm{x} 10^{9} / 1$ & 18 & $88.7 \pm 99.1$ & $119.5 \pm 81.1$ & 0.50 \\
\hline BM cellularity, $\%$ & 18 & $81.4 \pm 10.7$ & $64.5 \pm 27.3$ & 0.10 \\
\hline BM blasts, $\%$ & 18 & $13.1 \pm 5.9$ & $11.4 \pm 7.7$ & 0.60 \\
\hline Variable & $\mathrm{N}$ & $\mathrm{N}(\%)$ & $\mathrm{N}(\%)$ & P-value ${ }^{b}$ \\
\hline \multicolumn{5}{|l|}{ Sex } \\
\hline Male & 12 & $3(25)$ & $9(75)$ & \\
\hline Female & 6 & $4(66.7)$ & $2(33.3)$ & 0.08 \\
\hline \multicolumn{5}{|l|}{ MDS type } \\
\hline RCMD & 5 & $1(20)$ & $4(80)$ & \\
\hline RAEB-2 & 13 & $6(46.2)$ & $7(53.8)$ & 0.30 \\
\hline \multicolumn{5}{|c|}{ BM fibrosis WHO grade } \\
\hline MF-0 & 4 & $0(0)$ & $4(100)$ & \\
\hline MF-1 & 9 & $4(44.4)$ & $5(55.6)$ & 0.10 \\
\hline MF-2 & 5 & $3(60)$ & $2(40)$ & \\
\hline \multicolumn{5}{|l|}{ BM fibrosis pattern } \\
\hline Focal & 6 & $1(16.7)$ & $5(83.3)$ & \\
\hline Diffuse & 8 & $6(75)$ & $2(25)$ & 0.03 \\
\hline
\end{tabular}

(WBC) and platelet counts, percentage of CD34 positive blasts and MDS type; however, the rate of positive p53-IR was higher in patients with RAEB2 $(46.2 \%)$ than in those with RCMD (20\%). The results are summarized in Table I.

Associations between p53-IR, clinicopathological parameters and overall survival. At the time of analysis, 13 mortalities ( $72 \%$ of patients) had occurred, and 5 patients $(28 \%)$ were alive (censored). The mean OS for the whole series was 73 months (median, 18; range, 2-216). OS was significantly associated with patient age: At 5-year follow-up, 55\% of patients younger $<51$ years were alive compared to $44 \%$ of older patients $(\mathrm{P}=0.01)$. OS was also associated with hemoglobin level (5 year OS was $73 \%$ for patients with hemoglobin $>8 \mathrm{~g} / \mathrm{dl}$ vs. $14 \%$ for patients with hemoglobin $\leq 8 \mathrm{~g} / \mathrm{dl}$; $\mathrm{P}=0.04$ ), the type of MDS (5 year OS was $80 \%$ for patients with RCMD vs. $38 \%$ for patients with RAEB-2; $\mathrm{P}=0.05)$, the degree of BM fibrosis (Fig. 1A) (all patients without fibrosis were alive after 5 years vs. $34 \%$ of patients with WHO MF1/2; $\mathrm{P}=0.006$ ), the number of BM CD34-positive blasts (Fig. 1B) ( $80 \%$ of patients with $<5 \%$ BM blasts were alive after 5 years vs. $38 \%$ of those with $>5 \%$ BM blasts; $\mathrm{P}=0.05$ ). The median survival for patients with a negative p53-IR (Fig. 1D) was 105 months vs. 8 months for patients with a positive p53-IR
(Fig. 1C) $(\mathrm{P}=0.1)$. No difference in $\mathrm{OS}$ was found for sex, WBC and platelet counts. The results are summarized in Table II.

\section{Discussion}

Intense p53 (++) immunoreactivity was detected in $7 / 18$ cases $(38.8 \%)$, in line with the rate of positivity (44\%) previously reported in RCMD/RAEB2 cases (13) using similar staining and scoring procedure. Although previous studies used 1 or $2 \%$ of intensely immunostained nuclei as a cut off for p53 positivity $(11,17)$, the present study used $5 \%$ strongly $(++)$ immunopositive nuclei as a cut off, as it has been demonstrated that in $55 \operatorname{del}(5 \mathrm{q})$ patients, all those with $>5 \%$ p $53++$ stained cells in BM biopsies carried the TP53 mutation (11).

p53 immunopositivity was associated with BM fibrosis in the current study, in which no patients without BM fibrosis were p53-IR positive, whereas 50\% of those with WHO MF1-2 were, and $75 \%$ of patients with diffuse reticulin fibrosis were p53-IR positive, by contrast to only $16 \%$ of patients with focal fibrosis $(\mathrm{P}=0.03)$. Although no significant association was detected, p53 positivity was higher in RAEB2 than RCMD, in accordance with large studies revealing that TP53 mutations were observed mainly in patients with intermediate- 2 or high 
Table II. Correlation between clinical and histopathological bone marrow features and p53-IR with overall survival.

\begin{tabular}{|c|c|c|c|c|c|}
\hline Variable & $\mathrm{N}$ & $\begin{array}{l}\text { Median overall } \\
\text { survival, months }\end{array}$ & $\begin{array}{c}\text { 1-year overall } \\
\text { survival rate, \% }\end{array}$ & $\begin{array}{c}\text { 5-year overall } \\
\text { survival rate, \% }\end{array}$ & P-value ${ }^{a}$ \\
\hline Whole series & 18 & 18 & 61 & 49 & \\
\hline \multicolumn{6}{|l|}{ Sex } \\
\hline Male & 12 & 75 & 67 & 58 & \\
\hline Female & 6 & 8.2 & 50 & 33 & 0.60 \\
\hline \multicolumn{6}{|l|}{ Age, years } \\
\hline$\leq 51$ & 9 & 108 & 66 & 55 & \\
\hline$>51$ & 9 & 13 & 55 & 44 & 0.01 \\
\hline \multicolumn{6}{|l|}{ WBCs, $\times 10^{9} / 1$} \\
\hline$\leq 1.8$ & 5 & 12 & 60 & 40 & \\
\hline$>1.8$ & 11 & 9 & 55 & 44 & 0.70 \\
\hline \multicolumn{6}{|l|}{ Hb level, g/dl } \\
\hline$\leq 8$ & 7 & 8 & 42 & 14 & \\
\hline$>8$ & 11 & 105 & 73 & 73 & 0.04 \\
\hline \multicolumn{6}{|c|}{ Plt count, $\times 10^{9} / 1$} \\
\hline$\leq 100$ & 10 & 75 & 70 & 60 & \\
\hline$>100$ & 8 & 9 & 50 & 37 & 0.80 \\
\hline \multicolumn{6}{|l|}{ MDS type } \\
\hline RCMD & 5 & 105 & 100 & 80 & \\
\hline RAEB-2 & 13 & 12 & 46 & 38 & 0.05 \\
\hline \multicolumn{6}{|c|}{ BM fibrosis WHO grade } \\
\hline MF-0 & 4 & n.a. & 100 & 100 & \\
\hline $\mathrm{MF}-1 / 2$ & 14 & 12 & 57 & 34 & $<0.01$ \\
\hline \multicolumn{6}{|c|}{ BM fibrosis pattern } \\
\hline Focal & 6 & 18 & 67 & 50 & \\
\hline Diffuse & 8 & 8.2 & 37.5 & 25 & 0.20 \\
\hline \multicolumn{6}{|c|}{ BM CD34 positive blasts } \\
\hline$\leq 5$ & 5 & 105 & 100 & 80 & \\
\hline$>5$ & 13 & 12 & 46 & 38 & 0.05 \\
\hline \multicolumn{6}{|l|}{ P53-IR } \\
\hline Negative & 11 & 105 & 73 & 55 & \\
\hline Positive & 7 & 8 & 57 & 43 & 0.10 \\
\hline
\end{tabular}

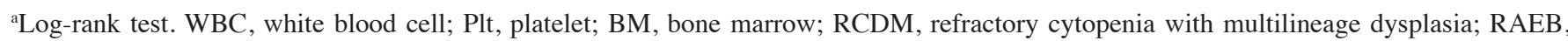
refractory anemia with excess blasts; p53-IR, p53 immunoreactivity; Hb, hemoglobin; MDS, myelodysplastic syndromes; WHO, World Health Organization; n.a., not applicable.

risk MDS (5). Therefore, with the limitation due to the number of cases, the current results indicate that a positive p53-IR at diagnosis is associated with adverse histological prognostic factors, such as BM fibrosis, and with clinically more aggressive MDS subtypes.

In addition, OS was associated with fibrosis in the present study; all patients without evidence of fibrosis in BM biopsy were alive at the 5-year follow-up, whereas only $34 \%$ of those with WHO MF-1/2 disease were $(\mathrm{P}=0.006)$. This finding is in agreement with large studies demonstrating the clinical relevance of bone marrow fibrosis in primary myelodysplastic syndromes $(18,19)$. OS was also associated with the type of MDS: $80 \%$ of patients with RCMD were alive at the 5-year follow-up, whereas only $38 \%$ of those with RAEB2 were $(\mathrm{P}=0.05)$.

Notably, in the present series, a positive p53-IR tended to be associated with a shorter OS: The median survival for patients with negative p53-IR was 105 months but only 8 months for those with positive $\mathrm{p} 53$-IR $(\mathrm{P}=0.1)$. This result is concordant with studies revealing the strong prognostic value of $\mathrm{p} 53$ mutations in MDS $(3,5,12,20)$. Mutations in TP53, enhancer of zeste homolog 2, ETS variant 6, runt-related transcription factor 1 and additional sex combs like 1 transcriptional regulator proteins were found to be predictors of poor overall survival in patients with MDS; however, only mutations in the TP53 gene have been clearly associated with poor prognostic 
markers, and have been reported to independently predict survival, primarily in patients with intermediate- 2 or high risk MDS (5). A large single institution study on 318 patients with MDS revealed that TP53 mutations were the strongest predictor of outcome in a multivariate model (12).

The present results emphasize the prognostic value of the immunohistochemical detection of p53 protein in MDS (11,14-17). The finding is particularly relevant, when considering the small number of cases and the type of therapy of the present series. All patients underwent allogeneic BM transplantation, by contrast to the previously reported series, which were primarily concerning patients with low- or intermediate risk del(5q) MDS treated with lenalidomide.

Immunohistochemistry is a practical and convenient method to detect p53 protein overexpression in tumor cells (6). An association has been reported between intense p53 nuclear staining and TP53 mutation in MDS $(11,13,17)$; furthermore, patients without TP53 mutations did not exhibit intense p53 protein staining, conferring a good negative predictive value for IHC (12). Therefore, the acceptable sensitivity of p53 immunostaining in predicting for TP53 mutations is encouraging, and the methodology is routine in diagnostic laboratories.

In conclusion, our findings suggest that the evaluation of p53-IR on BMBs of patients with MDS may be introduced in the histopathological work-up of the disease.

\section{Acknowledgements}

The present study was supported by grants from the Ministero Italiano dell'Università e Ricerca Scientifica (MIUR ex 60\%). This study was presented in part at the 19th World Congress on Advances in Oncology and 17th International Symposium on Molecular Medicine, 9-11 October 2014, Athens, Greece.

\section{References}

1. Brunning RD, Orazi A, Germing U, Le Beau MM, Porwit A, Bauman I, Vardiman JW and Hellstrom-Lindberg E: Myelodysplastic syndromes/neoplasms, overview. In: WHO Classification of Tumours of Haematopoietic and Lymphoid Tissues. Swerdlow SH, Campo E, Harris NL, Jaffe ES, Pileri S, Stein H, Thiele J and Vardiman JW (eds): 4th edition. IARC Press, Lyon, pp88-93, 2008.

2. Nagoshi H, Horiike S, Kuroda J and Taniwaki M: Cytogenetic and molecular abnormalities in myelodysplastic syndrome. Curr Mol Med 11: 678-685, 2011.

3. Kaneko H, Misawa S, Horiike S, Nakai H and Kashima K: TP53 mutations emerge at early phase of myelodysplastic syndrome and are associated with complex chromosomal abnormalities. Blood 85: 2189-2193, 1995.

4. Christiansen DH, Andersen MK and Pedersen-Bjergaard J: Mutations with loss of heterozygosity of p53 are common in therapy-related myelodysplasia and acute myeloid leukemia after exposure to alkylating agents and significantly associated with deletion or loss of $5 \mathrm{q}$, a complex karyotype and a poor prognosis. J Clin Oncol 19: 1405-1413, 2001.

5. Bejar R, Stevenson K, Abdel-Wahab O, Galili N, Nilsson B, Garcia-Manero G, Kantarjian H, Raza A, Levine RL, Neuberg D and Ebert BL: Clinical effect of point mutations in myelodysplastic syndromes. N Engl J Med 364: 2496-2506, 2011.
6. Kerns BM, Jordan PA, Moore MB, Humphrey PA, Berchuck A, Kohler MF, Bast RC Jr, Iglebart JD and Marks JR: p53 overexpression in formalin-fixed, paraffin-embedded tissue detected by immunohistochemistry. J Histochem Cytochem 40: 1047-1051, 1992.

7. Iggo R, Gatter K, Bartek J, Lane D and Harris AL: Increased expression of mutant forms of p53 oncogene in primary lung cancer. Lancet 335: 675-679, 1990.

8. Chang H, Yeung J, Qi C and Xu W: Aberrant nuclear p53 protein expression detected by immunohistochemistry is associated with hemizygous P53 deletion and poor survival for multiple myeloma. Br J Haematol 138: 324-329, 2007.

9. Chen MH, Qi CX, Saha MN and Chang H: p53 nuclear expression correlates with hemizygous TP53 deletion and predicts an adverse outcome for patients with relapsed/refractory multiple myeloma treated with lenalidomide. Am J Clin Pathol 137: 208-212, 2012

10. Chang H, Jiang AM and Qi CX: Aberrant nuclear p53 expression predicts hemizygous $17 p$ (TP53) deletion in chronic lymphocytic leukemia. Am J Clin Pathol 133: 70-74, 2010.

11. Jädersten M, Saft L, Smith A, Kulasekararaj A, Pomplun S, Göhring G, Hedlund A, Hast R, Schlegelberger B, Porwit A, et al: TP53 mutations in low-risk myelodysplastic syndromes with del(5q) predict disease progression. J Clin Oncol 29: 1971-1979, 2011.

12. Kulasekararaj AG, Smith AE, Mian SA, Mohamedali AM, Krishnamurthy P, Lea NC, Gäken J, Pennaneach C, Ireland R, Czepulkowski B, et al: TP53 mutations in myelodysplastic syndrome are strongly correlated with aberrations of chromosome 5, and correlate with adverse prognosis. Br J Haematol 160: 660-672, 2013

13. Müller-Thomas C, Rudelius M, Rondak IC, Haferlach T, Schanz J, Huberle C, Schmidt B, Blaser R, Kremer M, Peschel C, et al: Response to azacitidine is independent of p53 expression in higher-risk myelodysplastic syndromes and secondary acute myeloid leukemia. Haematologica 99: e179-e181, 2014.

14. Orazi A, Cattoretti G, Heerema NA, Sozzi G, John K and Neiman RS: Frequent p53 overexpression in therapy related myelodysplastic syndromes and acute myeloid leukemias: An immunohistochemical study of bone marrow biopsies. Mod Pathol 6: 521-525, 1993

15. Kitagawa M, Yoshida S, Kuwata T, Tanizawa T and Kamiyama R: p53 expression in myeloid cells of myelodysplastic syndromes. Association with evolution of overt leukemia. Am J Pathol 145: 338-444, 1994.

16. Brynes RK, Wilson CS, Kim AB and McCourty A: Expression of $\mathrm{p} 53, \mathrm{MDM} 2$, p21waf1, bcl-2, and retinoblastoma gene proteins in myelodysplastic syndrome after autologous bone marrow transplantation for lymphoma. Mod Pathol 10: 1120-1127, 1997.

17. Saft L, Karimi M, Ghaderi M, Matolcsy A, Mufti GJ, Kulasekararaj A, Göhring G, Giagounidis A, Selleslag D, Muus $\mathrm{P}$, et al: p53 protein expression independently predicts outcome in patients with lower-risk myelodysplastic syndromes with del(5q). Haematologica 99: 1041-1049, 2014.

18. Della Porta MG, Malcovati L, Boveri E, Travaglino E, Pietra D, Pascutto C, Passamonti F, Invernizzi R, Castello A, Magrini U, et al: Clinical relevance of bone marrow fibrosis and CD34-positive cell clusters in primary myelodysplastic syndromes. J Clin Oncol 27: 754-762, 2009.

19. Cazzola M and Malcovati L: Prognostic classification and risk assessment in myelodysplastic syndromes. Hematol Oncol Clin North Am 24: 459-468, 2010.

20. Sugimoto K, Hirano N, Toyoshima H, Chiba S, Mano H, Takaku F, Yazaki Y and Hirai H: Mutations of the $p 53$ gene in myelodysplastic syndrome (MDS) and MDS-derived leukemia. Blood 81: 3022-3026, 1993. 